\title{
Análisis psicométrico de la Escala de Autoconcepto AF5 de García y Musitu en estudiantes uniVersitarios de TARApoto (Perú)
}

\section{Psychometric analysis of Garcia and Musitu's AF5 Self-concept Scale on COLlege STUDENTS IN TARAPOTO (PeRÚ)}

\author{
Renzo Felipe Carranza Esteban* y Milton E. Bermúdez-Jaimes** \\ *Psicólogo y Magister en Educación. Docente en la Facultad de Ciencias de la Salud de la Universidad Peruana Unión \\ (UPeU). E-Mail: renzo.carranza@upeu.edu.pe \\ Jr. Los Mártires 218, San Martín-Morales, Perú. \\ **Doctor en Psicología. Docente Principal en la Facultad de Psicología de la Pontificia Universidad Javeriana. \\ E-Mail: milton.bermudez@javeriana.edu.co \\ Carrera 71D Nro. 97A-21 Torre 1 Apto 701, Colombia. \\ Universidad Peruana Unión (UPeU). Tarapoto - Perú. \\ Pontificia Universidad Javeriana. Bogotá - Colombia.
}

\section{Resumen}

El autoconcepto es uno de los factores que permite al ser humano gozar de salud y bienestar emocional durante su desarrollo evolutivo. Musitu, García y Gutiérrez (1991) mencionan que el autoconcepto es la percepción que tiene la persona de sí misma en base a la observación de sus capacidades y limitaciones y que le permite tener un sentido de la propia identidad. Quien posee un autoconcepto positivo, podrá ser capaz de aceptar retos, emprender desafíos, perder el temor, aventurarse y experimentar cosas nuevas. El estudio que se informa analiza las propiedades psicométricas de la Escala de Autoconcepto AF5 de García y Musitu. Se utilizó un diseño transversal con una muestra de 861 estudiantes de una universidad privada de Tarapoto (Perú). La escala es de tipo Likert y está compuesta por 30 ítems distribuidos en cinco dimensiones: autoconcepto académico, autoconcepto social, autoconcepto emocional, autoconcepto familiar y autoconcepto físico.

En primera instancia se realizó un análisis factorial exploratorio (AFE) con rotación Vari- max en el que cinco factores explicaron el 51.98\% de la variancia total. Posteriormente, se realizó con la técnica de ecuaciones estructurales un análisis factorial confirmatorio (AFC) para el cual se realizaron cuatro re-especificaciones. La solución final obtuvo índices de ajuste adecuados $(\mathrm{RMSEA}=.05 ; p=.05 ; \mathrm{TL} \mathrm{I}=.90 ; \mathrm{CFI}=.92$, $\mathrm{CMIN} / \mathrm{DF}=3.521 ; \mathrm{GFI}=.92$ y AGFI $=.90$ ). Entre los principales hallazgos se encontró que la solución final confirma la propuesta del autoconcepto como un constructo multidimensional de cinco factores. Sin embargo, el número de ítems difiere ligeramente. No se logró identificar una solución de segundo orden que evalúe el autoconcepto global. Los valores de confiabilidad para las escalas oscilaron entre .771 y .835. Los resultados obtenidos coinciden con los hallazgos de los autores originales por lo que se afirma que el AF5 es un instrumento válido y confiable para estudiantes universitarios peruanos.

Palabras clave: Autoconcepto; Estudiantes universitarios; Análisis factorial confirmatorio; Análisis factorial exploratorio; Validez; Confiabilidad, Propiedades psicométricas. 


\section{ABSTRACT}

Self-concept is one of the factors that allows humans to live healthy and with emotional wellbeing during their evolutionary development. $\mathrm{Mu}-$ situ, García, and Gutiérrez (1991) mention that selfconcept is the perception that people have of themselves based on the observation of their capabilities and limitations, which allows them to have a sense of identity. People having a positive self-concept may be able to accept dares, undertake challenges, lose fear, venture out, and try new things. In this regard, the present study analyzes the psychometric properties of García and Musitu's AF5 Selfconcept Scale. We used a cross-sectional design and the study sample consisted of 861 students of both genders (467 female and 394 male) in a private university in Tarapoto (Perú); who filled out the AF5 scale consisting of 30 items divided into five dimensions: academic self-concept, social self-concept, emotional self-concept, family self-concept, and physical self-concept, which are answered through a Likert scale.

Firstly, we calculated the Kaiser-Meyer-Olkin (KMO) sampling adequacy index for total scale where we obtained .885 and Bartlett's index of sphericity was $9377.994(d f=435, p=.001)$, these values allowed us to do the exploratory factor analysis (EFA) with Varimax rotation where five factors explained the $51.98 \%$ of the total variance. Later, a confirmatory factor analysis (CFA) was executed through the technique of structural equations for which four re-specification were done, the final solution got indexes of adequate adjustments (RMSEA $=.05, p=.05 ; \mathrm{TLI}=.90$, $\mathrm{CFI}=.92, \mathrm{CMIN} / \mathrm{DF}=3.521 ; \mathrm{GFI}=.92$, and AGFI $=.90$ ). Among the main findings we found that the final solution confirms the proposal of selfconcept as a multidimensional construct of five factors; however, the number of items differs slightly. It was not possible to identify a second order solution that may evaluate global self-concept. With respect to reliability, values for scales ranged from alpha equal to .771 and .835 . The results obtained coincide with the findings of the original authors; therefore, we state that the AF5 is a valid and reliable instrument for Peruvian university students.

The literature has shown that an adequate selfconcept is important in the life of every university student (Chen, Chiu, \& Wang, 2015; Jansen, Scherer, \& Schroeders, 2015; Zho, Ou \& Zhao, 2015). Taking into account that college entrance is usually the first major change in the life of a teenager, a phase where students tend to have certain academic conflicts, new living arrangements, academic pressure, new financial responsibilities, and stablishment of new relationships, both social and / or romantic, as a result of educational change; Therefore, if self-concept is not well defined, students will be more likely to show school desertion generated in the first years of higher education due to decompensated shock caused by the transition from high school routine life to an academic self-learning one. Chen, Chiu, and Wang (2015) found that self-concept has a positive and significant effect on a personal level and contributes to the development of the recognition of selflearning and meta-cognitive skills (to improve their academic performance). Jansen, Scherer, and Schroeders (2015) mention that self-concept is a better predictor of future on motivation to aspire to a career in science; and Zho, Ou, and Zhao (2015) emphasize that having a high self-concept, contributes to student performance, to make an effort to do homework or study, which allow to increase performance in college. Thus, in a university academic context, the literature reports that self-concept is an important factor for adequate adaptation to college life.

Key words: Self-concept; University students; Confirmatory factor analysis; Exploratory factor analysis; Validity; Reliability; Psychometric properties.

\section{INTRODUCCIÓN}

En la actualidad, el autoconcepto se ha convertido en un constructo muy estudiado por diferentes disciplinas, entre ellas la Psicología y la educación (Esnaola, Goñi \& Madariaga, 2008) y es definido como una fotografía que la persona posee de sí misma, de sus características, sus limitaciones y su capacidad potencial de interacción con los demás. Igualmente la literatura reporta que el desarrollo del autoconcepto será el que le 
permita al sujeto triunfar o fracasar (Enríquez Vereau, 1998). Por otra parte, Davidson y Neale (1991) han descripto el autoconcepto como un conjunto de percepciones organizado jerárquicamente, coherente y estable aunque también susceptible de cambios, que se construye por interacción a partir de las relaciones interpersonales.

La literatura señala que el autoconcepto no es unidimensional sino que por el contrario es multidimensional y estaría estructurado en varias áreas, tales como personal, académica, social y física (Shavelson, Hubner \& Stanton, 1976). Tomas y Oliver (2004) plantean que el autoconcepto

"es multidimensional ya que presenta diversos constructos o aspectos relacionados pero distinguibles" (p. 285).

En ese orden de ideas, la propuesta de $\mathrm{Mu}-$ sito, García y Gutiérrez (1991) sugiere que el autoconcepto está constituido por cinco dimensiones que trabajan de forma conexa e interrelacionada influyendo sustancialmente en su desarrollo y ellas son: académica, familiar, emocional, social y física. La investigación también señala que este constructo

“juega un papel decisivo y central en el desarrollo de la personalidad, tal como lo destacan las principales teorías psicológicas. Un autoconcepto positivo está en la base del buen funcionamiento personal, social y profesional dependiendo de él, en buena medida, la satisfacción personal, el sentirse bien consigo mismo" (Esnaola, Goñi \& Madariaga, 2008, p. 70).

$\mathrm{El}$ autoconcepto es un ente rector que resulta fundamental en la vida, se va formando en las diferentes etapas que atraviesa el ser humano (desde la niñez hasta la edad adulta) y donde el entorno social influye en su estructuración (Musito, García \& Gutiérrez, 1999). Asimismo, algunos estudios han reportado que se relaciona estrechamente con los problemas académicos, emocionales, con el bienestar personal (Casas et al., 2007; Diener, 1994; Furr \& Funder, 1998; Leung, J.P. \&
Leung, K., 1992; Martínez, Buelga \& Cava, 2007; Mruk, 2006), con el aprovechamiento académico (Henson \& Heller, 2000) y con el ajuste psicosocial en la adolescencia (Fuentes, García, Gracia \& Lila, 2011), por lo que este constructo sigue manteniendo su vigencia en la literatura especializada.

El interés por investigar el autoconcepto ha traído consigo la construcción de diversos instrumentos que lo evalúan. Muchos de ellos son traducciones de diferentes idiomas, en especial en lengua inglesa y aplicados sin considerar un análisis psicométrico robusto que permita evaluar su adecuación al contexto, o que al menos cuente con un proceso de adaptación o análisis de la pertinencia teórica de la prueba utilizada (Riquelme, E. \& Riquelme, P., 2011).

En este sentido, el instrumento con mayor uso internacional en lengua española es la Escala de Autoconcepto AF5, construida por García y Musitu $(1999,2001)$ para la población española y evalúa el autoconcepto global y específicamente en cinco de sus dimensiones: académico-laboral, social, emocional, familiar y física. Este instrumento está compuesto en total por 30 ítems, que se responden con una escala tipo Likert de cinco opciones que van desde nunca (1) hasta siempre (5). Los autores definen así a las dimensiones:

a.- el autoconcepto académico / laboral es la percepción que el estudiante tiene de la calidad de su desempeño, como educando y / o trabajador,

b.- el autoconcepto social es la percepción que el estudiante tiene de su desenvolvimiento en las relaciones sociales,

c.- el autoconcepto familiar se refiere a la percepción que tiene de su implicación, participación e integración en el ámbito familiar,

d.- el autoconcepto físico se refiere a la percepción que tiene la persona de su aspecto $\mathrm{y}$ de su condición física, y finalmente

e.- el autoconcepto emocional se refiere a la percepción que tiene de su estado emocional y sus respuestas a situaciones específicas, con cierto grado de compromiso e implicancia en su vida cotidiana (García \& Musitu, 1999, 2001). 
La muestra en la escala original estaba conformada por 6.483 participantes, de los cuales el $44 \%$ eran varones y el $56 \%$ mujeres, sus edades fluctuaban entre los 10 y los 62 años (García \& Musitu, 1999). Posteriores estudios psicométricos han reportado algunos análisis tanto de confiabilidad como de la estructura factorial de las versiones de la escala con distintas poblaciones. Algunos de estos reportes se sintetizan en la Tabla 1.

En el análisis de los reportes de investigación y con el objetivo de establecer criterios para definir el cumplimiento de los requisitos que den cuenta de la validez de constructo de un instrumento a través de la técnica de ecuaciones estructurales, se sugiere seguir los criterios propuestos por Byrne (2010) y Carretero-Dios y Pérez (2005). Estos criterios son una combinación de varios índices que garantizan la debida y adecuada interpretación de los resultados que señalan el cumplimento de la validez de constructo.

Dentro de esos índices destacados se consideran el valor del Índice de Bondad de Ajuste (GFI) y el Índice Ajustado de Bondad de Ajuste (AGFI) que debe ser mayor o igual a .90 para un ajuste óptimo; igualmente, con respecto al Índice de Ajuste Comparativo (CFI), Bentler (1995) sostiene que para aceptar el modelo propuesto su valor debe ser igual o superior a .90. En cuanto al error cuadrático medio de aproximación (RMSEA), si su valor es menor o igual a .05, el modelo presentará un buen ajuste y con respecto al Chi cuadrado, mientras más pequeño, mejor será el ajuste.

Tomando en consideración lo anterior, se puede concluir que solo los estudios de Riquelme, E. y Riquelme, P. (2011) para la población chilena y los de García-Grau, Ayora, Calabuig y Prado-Gascó (2014) con población infantil española, cumplen con todos los criterios. En los estudios de Tomás y Oliver (2004) y de Esnaola, Rodríguez y Goñi (2011) para población española universitaria, no alcanzan todos los criterios para un ajuste óptimo y algunos de los índices solo muestran un ajuste aceptable. Con respecto a la población peruana, Carranza (2012) realizó un análisis factorial exploratorio sobre este tema.
Por lo anterior y en consonancia con un vacío en la literatura, el propósito de este estudio fue analizar las propiedades psicométricas de la Escala de Autoconcepto AF5 de García y Musitu (1999) en una muestra de estudiantes universitarios de la ciudad de Tarapoto (Perú).

\section{MÉTOdo}

La investigación corresponde a un diseño no experimental de corte transversal y de tipo psicométrico (Cortada de Kohan, 1999).

\section{PARTICIPANTES}

La muestra estuvo compuesta por 861 estudiantes universitarios de primero a quinto año de todos los programas de formación de una universidad privada de Tarapoto; 467 eran mujeres y 394 varones $(54 \%$ y $46 \%$, respectivamente). El muestreo fue no probabilístico intencional y el reclutamiento de los participantes se hizo mediante convocatoria salón por salón, invitando a todos los estudiantes de la universidad. La edad promedio de los sujetos era 20 años $(D E=2.6$ años $)$. El 94\% de los participantes eran solteros, el $3.6 \%$ casados y un $2.4 \%$ reportaron otro estado civil. Con respecto al lugar de procedencia, el $64 \%$ era de la selva, $20.8 \%$ de la sierra, $13.2 \%$ de la costa y $1 \%$ del extranjero. Un $52.3 \%(n=450)$ de la muestra estuvo compuesta por estudiantes de la Facultad de Ciencias Empresariales, $26.2 \%(n=226)$ de la Facultad de Ingeniería y Arquitectura y un $21.5 \%(n=185)$ de la Facultad de Ciencias de la Salud. Inicialmente la muestra estuvo conformada por 943 estudiantes, sin embargo, el análisis final se realizó con 861datos pues se eliminaron los cuestionarios que estaban incompletos o mal diligenciados.

\section{INSTRUMENTO}

La AF5 puede ser aplicada a alumnos de $5^{\circ}$ y $6^{\circ}$ grados de primaria, secundaria y adultos. La confiabilidad del instrumento en su versión original es altamente significativa 
$(\alpha=.815)$ y su validez a través de la técnica de correlación demostró ser idónea $(r=.643)$. El manual técnico no reporta información acerca de la validez de constructo. La escala está compuesta por 30 ítems, distribuidos en cinco dimensiones con seis ítems cada una. La primera dimensión es autoconcepto académico / laboral, cuyos ítems son: 1, 6, 11, 16, 21 y 26 , la segunda dimensión es autoconcepto social y los ítems que le corresponden son: 2 , $7,12,17,22$ y 27 , la tercera dimensión es $a u$ toconcepto familiar cuyos ítems son: 29, 24, 19, 9, 14 y 4; la cuarta dimensión es el autoconcepto físico y los ítems que corresponden a esta son 10, 25, 20, 5, 30 y 15 y finalmente, la dimensión autoconcepto emocional, con los ítems 3, 8, 13, 18, 23 y 28. Para este estudio se utilizó la versión de Musitu, García y Gutiérrez (1991).

\section{Procedimiento}

Se solicitó la autorización del comité de ética institucional y de los docentes de cada aula de clases, como así también la participación voluntaria de los alumnos, bajo la condición de respetar su privacidad y confidencialidad de la información. Se les informaron los fines del estudio, entregándoles un ejemplar del instrumento impreso con un lapicero y se los orientó sobre el contenido, propósito y modo de respuestas a los ítems del instrumento, con un tiempo estimado de 15 minutos para su desarrollo. La administración de la prueba se realizó durante el período de clases.

\section{ANÁLISIS DE DATOS}

Se realizó en tres pasos: análisis factorial exploratorio, análisis factorial confirmatorio y análisis de consistencia interna. En primera instancia, se exploró la base de datos para identificar datos perdidos en las respuestas de los participantes, los cuales fueron eliminados de la base final. Luego se procedió a evaluar si el tamaño de la muestra presentaba un adecuado ajuste para realizar el análisis factorial; para ello se utilizaron dos estadísticos, el Kaiser-Meyer-Olkin y la prueba de es- fericidad de Bartlett, también se examinaron los valores de extracción de las comunidades y el porcentaje total de variancia explicada. Esta fase del análisis factorial exploratorio (AFE) concluyó con una solución forzada a cinco factores como originalmente lo proponen los autores García y Musitu (1999). La solución se rotó por la técnica VARIMAX debido a que se supone una solución ortogonal de factores independientes. Todos estos procedimientos se llevaron a cabo con el software de análisis estadístico SPSS $^{\circledR}$ versión 22 , estableciéndose un nivel de significción igual a $.05(5 \%)$.

A continuación se realizó el análisis factorial confirmatorio mediante la técnica de ecuaciones estructurales como lo sugieren Byrne (2010) y Kline (2010), con el software AMOS 22 y se realizó con el siguiente procedimiento: se modeló la solución factorial del AFE y se evaluaron los siguientes índices de bondad de ajuste: El Chi cuadrado de ajuste absoluto / predictivo, el TLI (Índice Tucker-Lewis), CFI (Índice de Ajuste Comparativo), el GFI (Índice de Bondad de Ajuste), el AGFI (Índice de Bondad de Ajuste Corregido) y el RMSEA (Raíz Media Cuadrática Estandarizada).

Posteriormente, se realizaron varias reespecificaciones del modelo utilizando covariancias de error a partir de los índices de modificación propuestos hasta alcanzar el ajuste adecuado según el criterio de Byrne (2010). Para finalizar se recalculó la confiabilidad de las cinco escalas mediante la técnica de consistencia interna usando el estadístico Alpha de Cronbach para cumplir con los estándares de un instrumento de evaluación psicológica (Mikulic, Crespi \& Radusky, 2015).

\section{Resultados}

\section{ANÁlisis FACTORIAL EXPLORATORIO (AFE)}

El índice de adecuación muestral KaiserMeyer-Olkin (KMO) para la escala total fue igual a .885 y el índice de esfericidad de Bartlett fue igual a $9377.994(\mathrm{gl}=435$; 
$p=.001)$, estos valores indican un adecuado ajuste de la muestra para poder realizar el análisis factorial. Asumiendo de manera $a$ priori la estructura interna de la prueba en sus cinco dimensiones: académica, emocional, familiar, social y física, se realizó un análisis factorial de componentes principales con rotación VARIMAX. Los resultados se presentan en la Tabla 2.

La solución rotada a cinco componentes explica una variancia total de $51.9 \%$ para 25 ítems, es decir, 5 de los ítems originales no cumplieron con los requisitos de carga factorial para la solución original propuesta por los autores. Los ítems eliminados de esta solución fueron los siguientes: P5, P15, P20, P22 y $\mathrm{P} 30$, cuatro de ellos de la dimensión del Autoconcepto físico. Las comunalidades oscilaron entre .379 para el ítem P11 y .767 para el ítem P25.

\section{AnÁlisis Factorial ConfiRmatorio (AFC)}

Para probar la validez de constructo se utilizó la técnica de ecuaciones estructurales que sugieren Kline (2010) y Byrne (2010). En primer lugar, la solución del AFE se modeló en el Programa AMOS 22 y se calcularon los valores estadísticos para estimados estandarizados, momentos residuales, cargas factoriales, covariancias y correlaciones estimadas e índices de modificación. La solución original no obtuvo los índices de ajuste requeridos por lo que se procedió a realizar cuatro re-especificaciones del modelo utilizando los índices de modificación correlacionando los errores de ítems de los mismos factores (ver Tabla 3).

En la primera re-especificación se establecieron tres interacciones de errores estandarizados entre los ítems P1 y P14, P16 y P21, P14 y P24 y se realizó un nuevo análisis.

En la segunda re-especificación se establecieron dos interacciones de errores estandarizados entre los ítems P4 y P9 y P1 y P6.

En la tercera re-especificación se establecieron dos interacciones de errores estandarizados entre los ítems P12 y P2, P18 y P23. $Y$ finalmente, en la cuarta re-especificación se establecieron dos interacciones de errores estandarizados entre los ítems P11 y P6, P11 y P1 obteniendo el ajuste adecuado del model $\left(\chi^{2}=869.666 ; g l=247 ; p<.001\right.$, $\mathrm{TLI}=.90 ; \mathrm{CFI}=.92 ; \mathrm{GFI}=.92 ; \mathrm{AGFI}=.90$ y RMSEA = .054). En la Figura 1 se observa la solución final.

Los valores de confiabilidad Alpha para las escalas fueron los siguientes: Académica .835, Emocional .790, Familiar .782, Social .792 y Física .771. Es importante indicar que un factor de segundo orden que evaluaría un autoconcepto global no se pudo verificar.

\section{DISCUSIÓN}

El objetivo del estudio fue analizar las propiedades psicométricas de la Escala de Autoconcepto AF5 de García y Musitu en estudiantes universitarios de Tarapoto (Perú). Una de las contribuciones de mucha importancia que realiza el estudio que se informa fue corroborar que el AF5 cumple con los requisitos de validez y confiabilidad similares a los reportados originalmente por los autores de la prueba. Otra contribución es la de proveer un instrumento válido y confiable para universitarios peruanos.

Debido al auge que ha tenido la investigación sobre el autoconcepto, los investigadores necesitan contar con instrumentos que cumplan con todos los requisitos psicométricos que garanticen la calidad de los resultados que proporcionan sus estudios. Si bien se han adelantado diversos estudios dirigidos a contar con este tipo de instrumentos, en el reporte de la literatura sobre adaptaciones culturalmente válidas hay diversos hallazgos por lo que aún se requiere contar con mucha evidencia que garantice evaluaciones confiables y válidas. En concreto, con respecto al instrumento AF5 se dispone de varios estudios psicométricos con resultados mezclados que confirman en algunos casos la estructura factorial original (Riquelme, E. \& Riquelme, P. 2011) y otros encuentran estructuras factoriales distintas (Malo, Bataller, Casas, Gras \& Gonzáles, 2011). 
En el análisis factorial exploratorio realizado para establecer el número mínimo de factores capaces de reproducir de manera satisfactoria, las correlaciones entre los 30 ítems del AF5 mostraron una estructura de cinco factores de autoconcepto: académico, social, familiar, físico y personal, que coincide con los autores originales de la prueba (García \& Musitu, 1999) y que se asemejan a lo encontrado en muestras de Italia (Marchetti, 1997), Brasil (Martínez \& García, 2008; Martínez, García \& Yubero, 2007; Martínez, Musitu, García \& Camino, 2003) y Portugal (García, Musitu \& Veiga, 2006). Entonces, resulta importante destacar que la Escala de Autoconcepto AF5 de García y Musitu para universitarios peruanos presenta evidencias de validez de constructo.

El análisis factorial confirmatorio indicó que el modelo de medición de 30 ítems en cinco factores se ajusta parcialmente al modelo teórico, pues solo dos ítems del factor físico se mantienen en la versión final de la escala.

Con respecto a la confiabilidad del instrumento, se aprecia que la escala presenta una muy buena confiabilidad, los índices de consistencia interna estimada mediante el coeficiente Alpha de Cronbach son altos (.866), pues los valores estimados superan el umbral del punto de corte igual a .75 , considerado como indicador de una buena confiabilidad para los instrumentos de medición psicológica (Polit \& Hungler, 2013; Rodríguez de Behrends \& Brenlla, 2015).

Pese a lo anterior, es importante señalar algunas limitaciones que se encuentran en el estudio realizado. No se utilizaron medidas para evaluar la validez externa del AF5. Asimismo, en futuras investigaciones es necesario analizar la dimensión física del autoconcepto pues como se pudo apreciar, solo 2 de los 6 ítems originales se mantienen y dada la diversidad geográfica del Perú, es necesario tomar muestras en otras regiones del país, como la costera, sierra y otros lugares de la selva, en donde se ha evidenciado diferencias en algunos constructos psicológicos y educativos (Escurra et al., 2001; León, 2001). También es importante señalar que se pudo haber presentado un sesgo de selección en la conformación de la muestra porque el procedimiento de reclutamiento no fue aleatorio sino por conveniencia.

En ese sentido, este estudio sugiere contar con muestras representativas aleatorias como así también con el desarrollo de investigaciones que analicen la validez externa y concurrente a fin de garantizar más efectivamente las propiedades psicométricas del AF5. También sería importante trabajar con otros constructos tales como autoestima, autoeficacia, autoconocimiento, motivación, optimismo, de modo que de darle una mayor comprensión al constructo estudiado.

A pesar de las limitaciones, se considera que la escala AF5 de García y Musitu es válida y confiable para estudiantes universitarios de Tarapoto, asimismo contribuye al desarrollo de investigaciones sobre el autoconcepto en la etapa preescolar, escolar, adolescencia y juventud. No obstante, puede contribuir en otros contextos (áreas de la Psicología) poco estudiados tales como organizacional, clínico $\mathrm{y}$ forense. 


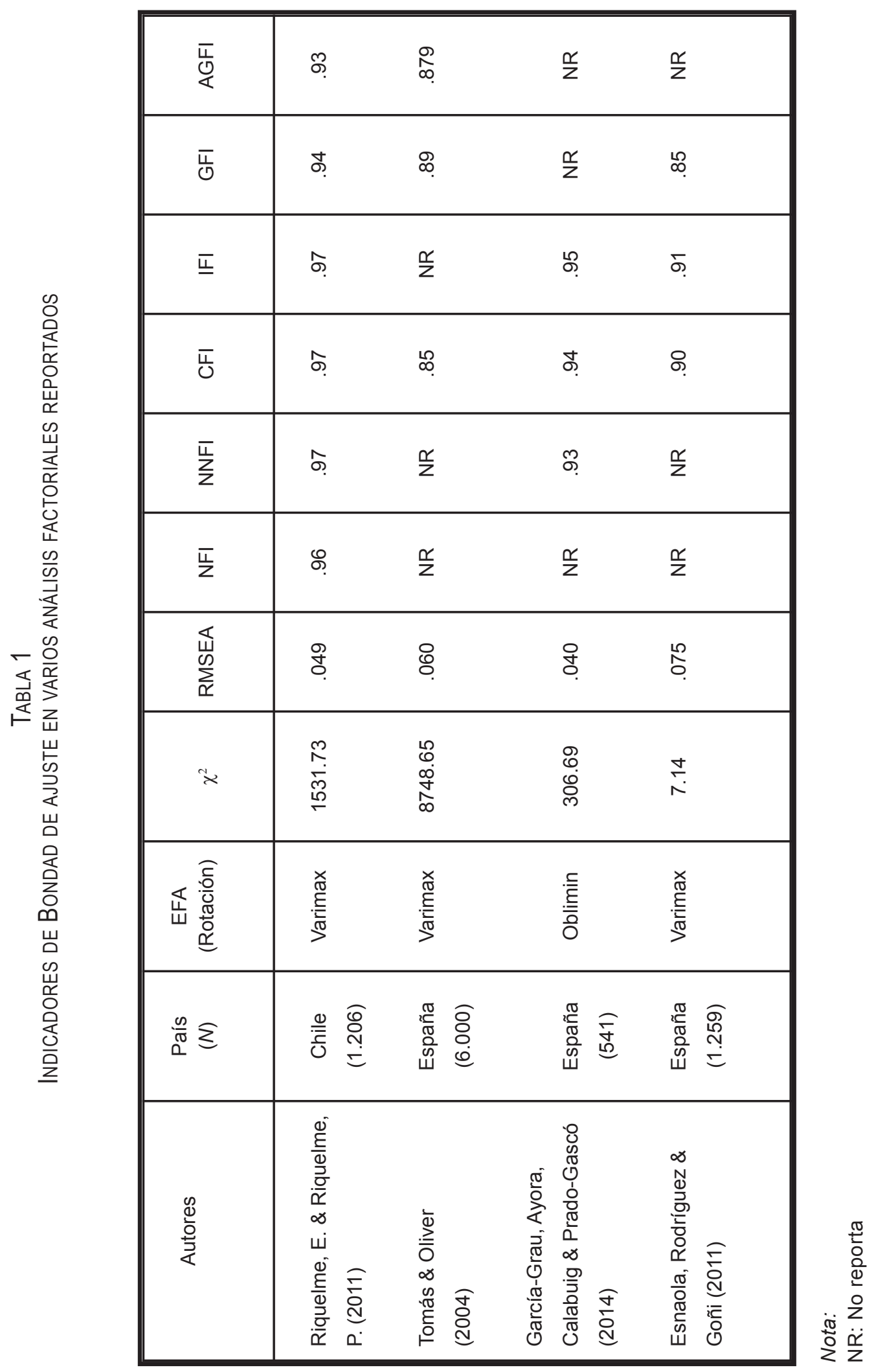




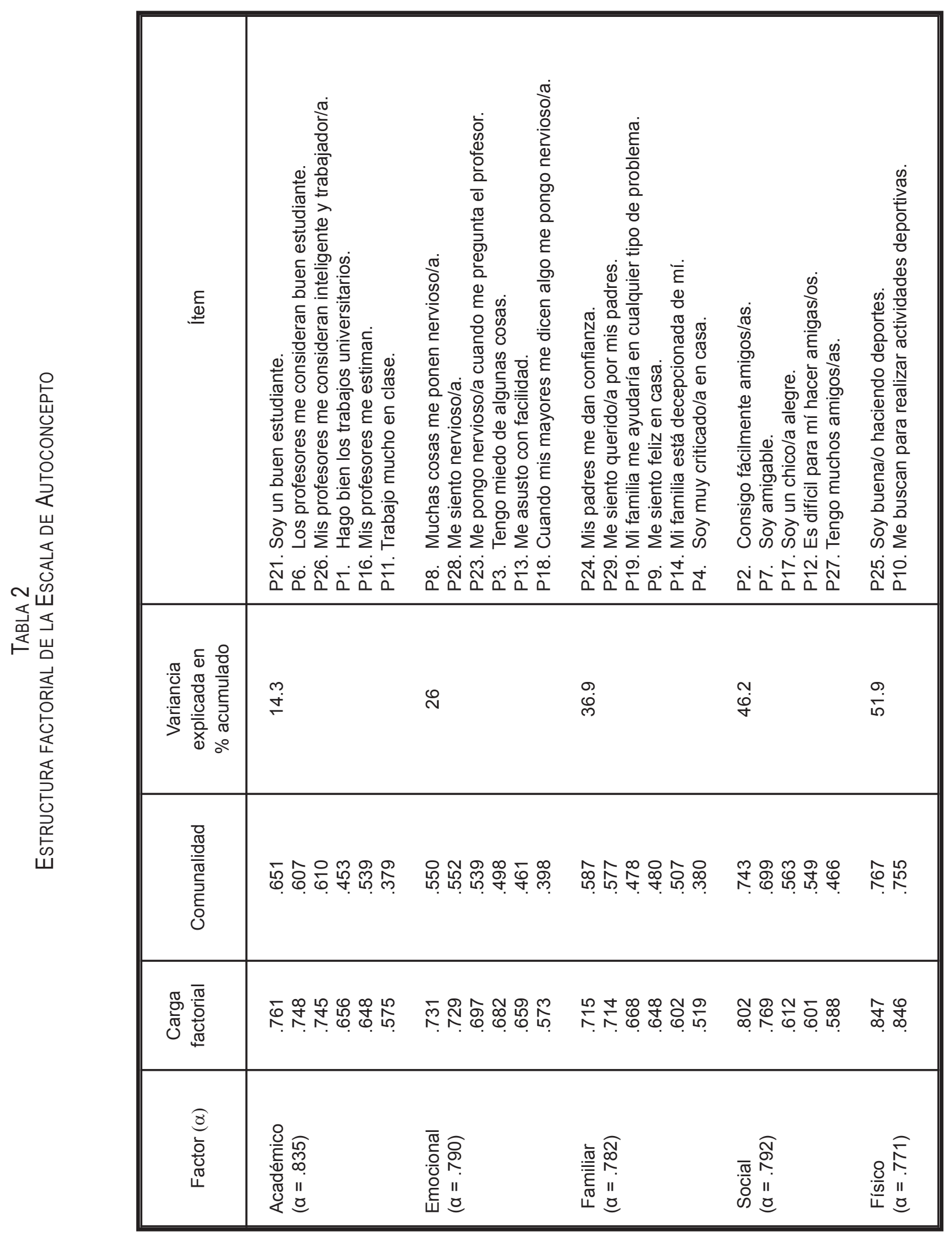




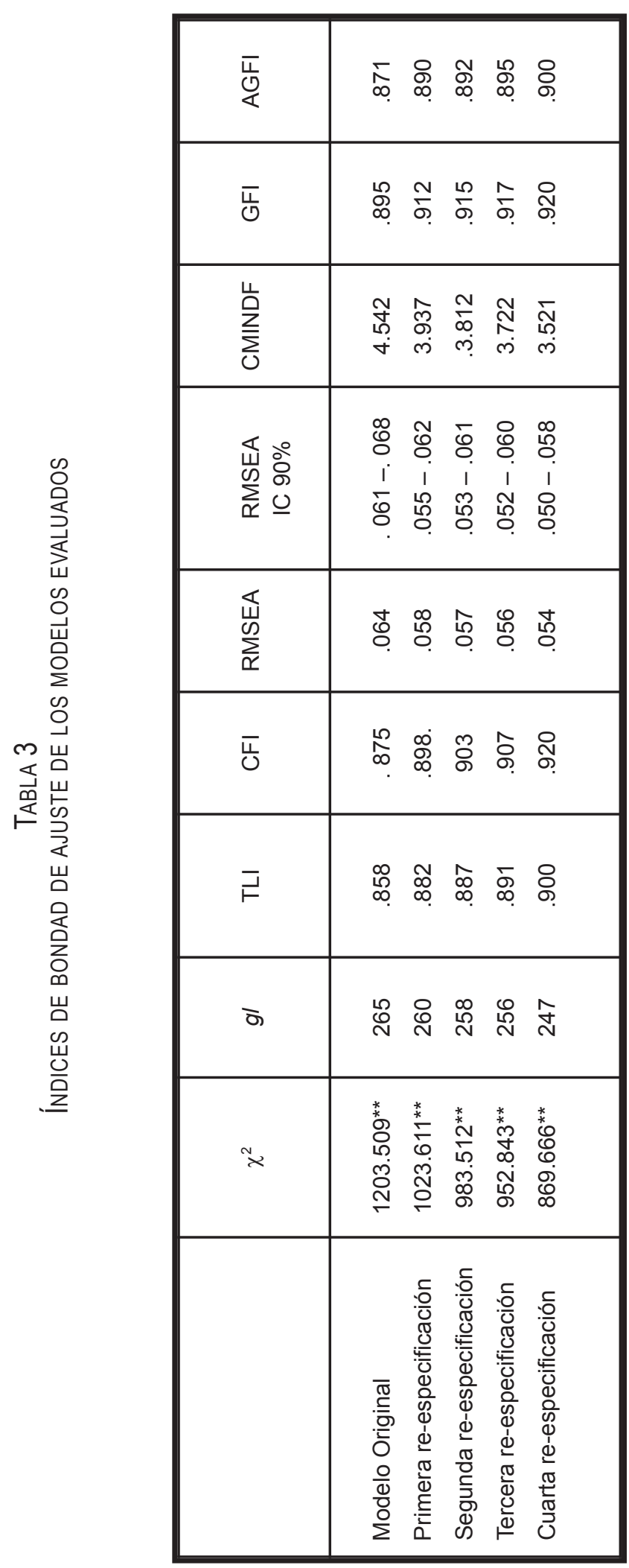

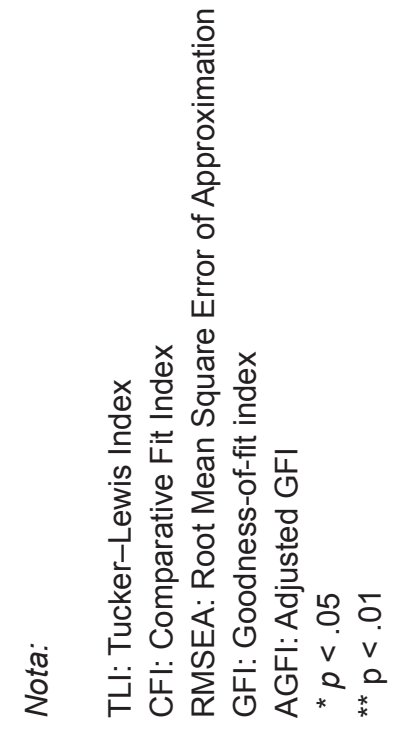


FIGURA 1

AnÁlisis Confirmatorio de la Escala AF5 de García y Musitu $(1999,2001)$

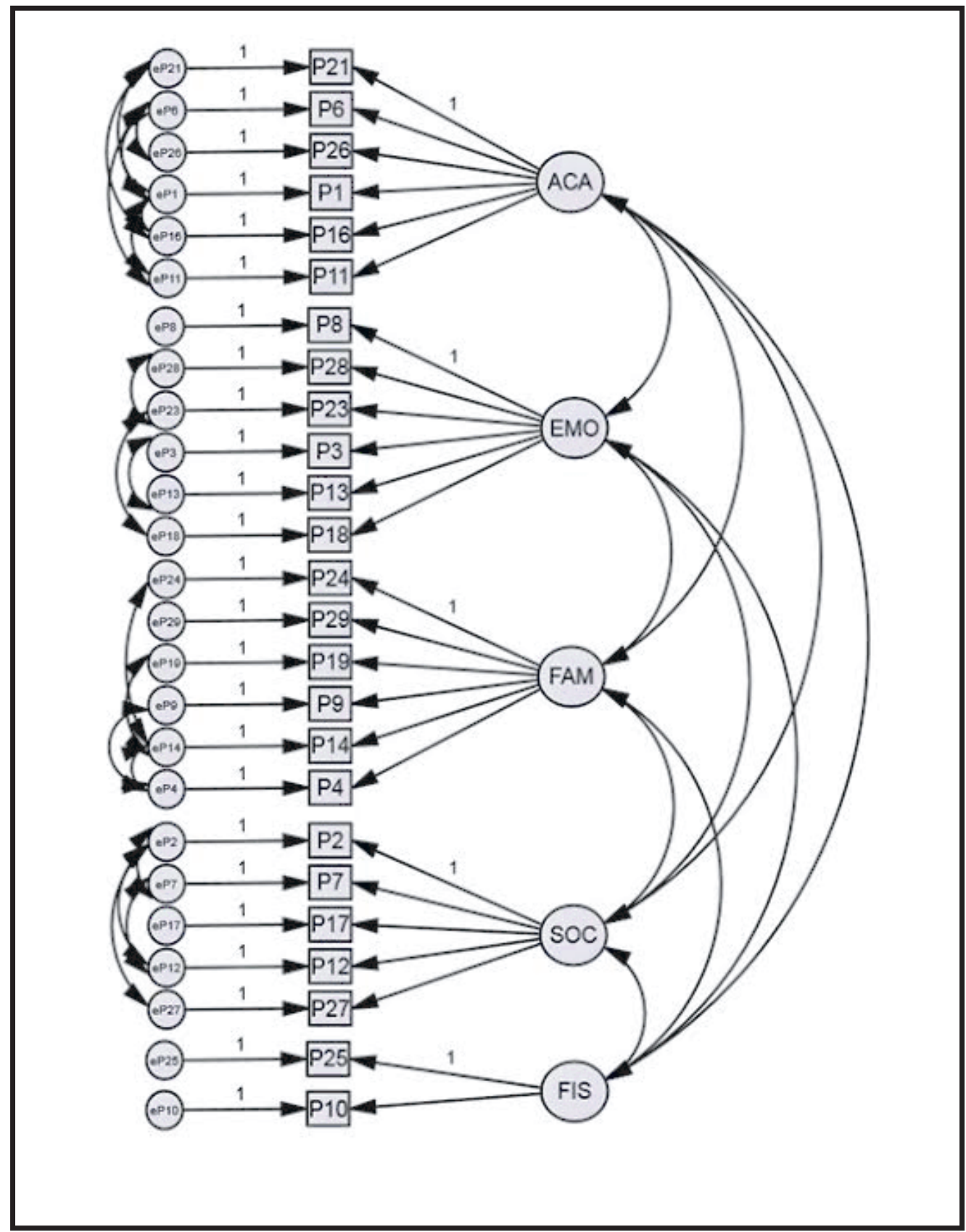




\section{REFERENCIAS BIBLIOGRÁFICAS}

Bentler, P.M. (1995). EQS Structural Equation Program Manual. Encino, CA: Multivariate Software.

Byrne, B.M. (2010). Structural equation modeling with EQS: Basic concepts, applications, and programming (2da. ed.). Mahwah, NJ: Erlbaum.

Carranza, P. (2012). Propiedades psicométricas del Cuestionario de Autoconcepto Forma 5 en estudiantes de secundaria del Distrito de Moche [Psychometric properties of Self-concept Questionnaire Form 5 on high school students in Moche District]. Tesis de Licenciatura no publicada. Universidad César Vallejo. Trujillo, Perú. Recuperado el 19 de enero de 2016 de http: //es.scribd.com/doc/152014922/Tesis-Af5-Ped ro-Sustentar\#scribd

Carretero-Dios, H. \& Pérez, C. (2005). Normas para el desarrollo y revisión de estudios instrumentales [Norms for the development and revision of instrumental studies]. International Journal of Clinical and Health Psychology, 5, 521-551.

Casas, F., Figuer, C., González, M., Malo, S., Alsinet, C. \& Subarroca, S. (2007). The well-being of 12- to 16-year-old adolescents and their parents: Results from 1999 to 2003 Spanish samples. Social Indicators Research, 83(1), 87-115.

Chen, B.H., Chiu, W. \& Wang, C. (2015). The relationship among academic self-concept, learning strategies, and academic achievement: A case study of National Vocational College Students in Taiwan via SEM. Asia-Pacific Education Researcher, 24(2), 419-431. https://doi.org/10. 1007/s40299-014-0194-1

Cortada de Kohan, N. (1999). Teorías psicométricas y construcción de tests [Psychometric theories and tests construction]. Buenos Aires: Lugar Editorial.
Davidson, G. \& Neale, J.M. (1991). Abnormal psychology. Nueva York: John Wiley.

Diener, E. (1994). El bienestar subjetivo [Subjective well-being]. Intervención Psicosocial, 3(8), 67-113.

Enríquez Vereau, J. (1998). El autoconcepto y la ansiedad ante los exámenes como predictores del rendimiento académico en estudiantes de secundaria de un colegio nacional de La Moli$n a$ [Self-concept and the anxiety as predictors of academic performance on high school students in a national school in La Molina]. Tesis de Licenciatura no publicada. Universidad Inca Garcilaso de la Vega, Lima, Perú.

Escurra. L., Delgado, A., Quesada, R., Rivera, J., Santos, J., Rivas, G. \& Pequeña, J. (2001). Construcción de una prueba de inteligencia emocional [Construction of an emotional intelligence test]. Revista de Investigación en Psicología del Instituto de Investigaciones Psicológicas, 4(2), 83-95.

Esnaola, I., Goñi, A. \& Madariaga, J. (2008). El autoconcepto: Perspectivas de investigación [Selfconcept: Research perspectives]. Revista de Psicodidáctica, 13(1), 179-194.

Esnaola, I., Rodríguez, A. \& Goñi, E. (2011). Propiedades psicométricas del Cuestionario de $\mathrm{Au}$ toconcepto AF5 [Psychometric properties of Self-concept Questionnaire AF5]. Anales de Psicología, 27(1), 109-117.

Fuentes, M., García, J., Gracia, E. \& Lila, M. (2011). Autoconcepto y ajuste psicosocial en la adolescencia [Self-concept and psychosocial adjustment in adolescence]. Psicothema, 23(1), 7-12.

Furr, R.M. \& Funder, D. (1998). A multimodal analysis of personal negativity. Journal of Personality and Social Psychology, 74(6), 15801591.

García-Grau, P., Ayora, D. \& Calabuig, F. (2014). Autoconcepto en la preadolescencia: Una versión breve de la Escala AF5 [Self-concept in 
preadolescence: A brief version of the AF5 scale]. Revista de Educação Física, 20(2), 151157. http://dx.doi.org/10.1590/S1980-657420 14000200004

García, F. \& Musitu, G. (1999). AF5, Autoconcepto Forma 5 [SF5, Self-concept Form 5]. Madrid: TEA

García, F. \& Musitu, G. (2001). AF5, Autoconcepto Forma 5 - Segunda edición [SF5, Self-concept Form 5 - Second edition]. Madrid: TEA.

García, J.F., Musitu, G. \& Veiga, F. (2006). Autoconcepto en adultos de España y Portugal [Selfconcept in adults from Spain and Portugal]. Psicothema, 18(3), 551-556.

Henson, K. \& Heller, B. (2000). Psicología educativa en la enseñanza eficaz [Educational psychology in effective teaching]. México: Internacional Thompson Editores México.

Jansen, M., Scherer, M. \& Schroeders, U. (2015). Students' self-concept and self-efficacy in the sciences: Differential relations to antecedents and educational outcomes. Contemporary Educational Psychology, 41, 13-24. Recuperado el 20 de febrero de 2016 de http://ezproxy.averia na.edu.co:2143/full_record.do?product=UA\&s earch $\_$mode $=$GeneralSearch\&qid $=1 \& \mathrm{SID}=1 \mathrm{~A}$ FvcvFWYCgQbIC67Mj\&page $=1 \&$ doc $=7 \& \mathrm{ca}$ cheurlFromRightClick=no

Kline, R.B. (2010). Principles and practice of structural equation modelling. NY: Guilford.

León, C. (2001). La inteligencia emocional en estudiantes de educación superior universitaria y no universitaria de administración de empresas [Emotional intelligence in students of university and non-university higher education of business administration]. Tesis de Licenciatura no publicada. Universidad Inca Garcilaso de la Vega, Lima.

Leung, J.P. \& Leung, K. (1992). Life satisfaction, self-concept and relationship with parents in adolescent. Journal of Youth and Adolescence, 21(6), 653-665.
Malo, S., Bataller, S., Casas, F., Gras, E. \& Gonzáles, M. (2011). Análisis psicométrico de la Escala Multidimensional de Auto-concepto AF5 en una muestra de adolescentes y adultos de Cataluña [Psychometric analysis of the SF5 multidimensional scale of self-concept in a sample of adolescents and adults in Catalonia]. Psicothema, 23(4), 871-878. Recuperado el 20 de mayo de 2016 de http://www.psicothema.com/ pdf/3969.pdf

Marchetti, B. (1997). Concetto di se'relazioni familiari e valori. Tesis de Licenciatura no publicada. Universita degli Studi di Bologna, Bologna, Italia.

Martínez, M., Buelga, S. \& Cava, M.J. (2007). La satisfacción con la vida en la adolescencia y su relación con la autoestima y el ajuste escolar [Satisfaction with life in adolescence and its relationship to self-esteem and school adjustment]. Anuario de Psicología, 38(2), 5-15.

Martínez, I. \& García, J.F. (2008). Internalization of values and self-esteem among Brazilian teenagers from authoritative, indulgent, authoritarian, and neglectful home. Adolescence, 43 (169), 13-29.

Martínez, I., García, J.F. \& Yubero, S. (2007). Parenting styles and adolescents self-esteem in Brazil. Psychological Reports, 100(3), 731-745.

Martínez, J., Musitu, G., García, J.F. \& Camino, L. (2003). Un análisis transcultural de los efectos de la socialización familiar en el autoconcepto: España y Brasil [A transcultural analysis of the effects of family socialization in self-concept: Spain and Brazil]. Psicología, Educaçâo e Cultura, 2(2), 239-258

Mikulic, I.M., Crespi, M. \& Radusky, P. (2015). Construcción y validación del Inventario de Competencias Socioemocionales para adultos (ICSE) [Construction and validation of Socioemotional Competence Inventory for Adults (SECI)]. Interdisciplinaria, 32(2), 307-329. Recuperado el 10 de octubre de 2016 de http:// 
4www.redalyc.org/articulo.oa?id $=1804352800$ https://doi.org/10.16888/interd.2015.32.2.7

Mruk, C.J. (2006). Self-esteem research, theory and practice: Toward a positive psychology of self-esteem. New York: Springer.

Musitu, G., García, F. \& Gutiérrez, M. (1991). AF A, Autoconcepto Forma A [SFA, Self-concept Form A]. Madrid, España: TEA.

Musitu, G., García, F. \& Gutiérrez, M. (1999). Autoconcepto Forma A. Manual [Self-concept Form A. Manual]. Madrid: TEA.

Polit, D.F. \& Hungler, B.P. (2013). Essentials of nursing research: Methods, appraisal, and utilization (8va. ed.). Philadelphia: Wolters Kluwer.

Riquelme, E. \& Riquelme, P. (2011). Análisis psicométrico confirmatorio de la medida multidimensional del Test de Autoconcepto Forma 5 en Español (AF5), en estudiantes universitarios de Chile [Confirmatory psychometric analysis of multidimensional scale of the Self-concept test form 5 in Spanish (AF5), in university students in Chile]. Psicologia, Saúde \& Doenças, 12(1), 91-103. Recuperado el 30 de marzo de 2016 de http://www.scielo.gpea ri.mctes. pt/pdf/psd/v12n1/v12n1a06.pdf
Rodríguez de Behrends, M. \& Brenlla, M.E. (2015). Adaptación para Buenos Aires de la Escala de Intolerancia a la Incertidumbre [Adaptation for Buenos Aires of the intolerance of uncertainty scale]. Interdisciplinaria, 32(2), 261-274. Recuperado el 10 de octubre de 2016 de http://4ww w.redalyc.org/articulo.oa?id=18043528004

Shavelson, R., Hubner, J. \& Stanton, J. (1976). Self concept: Validation of construct interpretations. Review of Educational Research, 46(3), 407-441.

Tomás, J.M. \& Oliver, A. (2004). Análisis psicométrico confirmatorio de una medida multidimensional de autoconcepto en español [Confirmatory psychometric analysis of a multidimensional Scale of Self-concept in Spanish]. Revista Interamericana de Psicología, 38(2), 285293.

Zho, Y., Ou, C. \& Zhao, Z. (2015). The impac of self-concept and cllege involvent on the firstyears success of medicalstudents in China. Advances in Healt Sciences Education, 20(1), 163-179. https://doi.org/10.1007/s10459-0149515-7
Universidad Peruana Unión (UPeU)

Tarapoto - Perú

Pontificia Universidad Javeriana

Bogotá - Colombia

Fecha de recepción: 22 de agosto de 2016 Fecha de aceptación: 24 de enero de 2017 